\title{
Does urinary peptide content differ between COPD patients with and without inherited alpha-I antitrypsin deficiency?
}

This article was published in the following Dove Press journal:

International Journal of COPD

8 March 2017

Number of times this article has been viewed

\author{
Alfonso Carleo ${ }^{1,2, *}$ \\ Joanna Chorostowska- \\ Wynimko ${ }^{3, *}$ \\ Thomas Koeck ${ }^{4}$ \\ Harald Mischak ${ }^{4,5}$ \\ Małgorzata Czajkowska- \\ Malinowska ${ }^{6}$ \\ Adriana Rozy ${ }^{3}$ \\ Tobias Welte ${ }^{1,2}$ \\ Sabina Janciauskiene ${ }^{1,2}$ \\ 'Department of Respiratory \\ Medicine, Hannover Medical School, \\ ${ }^{2}$ Biomedical Research in Endstage \\ and Obstructive Lung Disease \\ Hannover (BREATH), DZL Hannover, \\ Germany; ${ }^{3}$ Department of Genetics \\ and Clinical Immunology, National \\ Institute of Tuberculosis and Lung \\ Diseases, Warsaw, Poland; ${ }^{4}$ Mosaiques \\ Diagnostics and Therapeutics AG, \\ Hannover, Germany; ${ }^{5}$ Institute of \\ Cardiovascular and Medical Sciences, \\ University of Glasgow, Glasgow, UK; \\ ${ }^{6}$ Department of Lung Diseases and \\ Respiratory Failure, Regional Center \\ of Pulmonology, Bydgoszcz, Poland \\ *These authors contributed equally \\ to this work
}

Correspondence: Sabina Janciauskiene Department of Respiratory Medicine, Hannover Medical School, Feodor-Lynen Str 23, 30625 Hannover, Germany

Tel +49 5II 5327297

Fax +49 5II 5327294

Email janciauskiene.sabina@

mh-hannover.de
Abstract: Differentiating between chronic obstructive pulmonary disease (COPD) patients with normal (PiMM) or deficient (PiZZ) genetic variants of alpha-1 antitrypsin (A1AT) is important not only for understanding the pathobiology of disease progression but also for improving personalized therapies. This pilot study aimed to investigate whether urinary peptides reflect the A1AT-related phenotypes of COPD. Urine samples from 19 clinically stable COPD cases (7 PiMM and 12 PiZZ A1AT) were analyzed by capillary electrophoresis coupled to mass spectrometry. We identified 66 peptides (corresponding to 36 unique proteins) that differed between PiZZ and PiMM COPD. Among these, peptides from the collagen family were the most abundant and divergent. A logistic regression model based on COL1A1 or COL5A3 peptides enabled differentiation between PiMM and PiZZ groups, with a sensitivity of $100 \%$ and specificity of $85.71 \%$ for COL1A1 and a sensitivity of $91.67 \%$ and specificity of $85.71 \%$ for COL5A3. Furthermore, patients with PiZZ presented low levels of urinary peptides involved in lipoproteins/lipids and retinoic acid metabolism, such as apolipoprotein A-I and C4, retinol-binding protein 4 and prostaglandin- $\mathrm{H} 2$ D-isomerase. However, peptides of MDS1 and EVII complex locus, gelsolin and hemoglobin alpha were found in the urine of COPD cases with PiZZ, but not with PiMM. These capillary electrophoresis coupled to mass spectrometry-based results provide the first evidence that urinary peptide content differs between PiMM and PiZZ patients with COPD.

Keywords: alpha-1 antitrypsin, alpha-1 antitrypsin deficiency, COPD, urine, peptidomics, capillary electrophoresis coupled to mass spectrometry, phenotypes, peptides, biomarkers, collagen

\section{Introduction}

Chronic obstructive pulmonary disease (COPD), characterized by the chronic and progressive obstruction of lung airflow, ${ }^{1}$ is the fourth leading cause of mortality and morbidity worldwide. ${ }^{2}$ COPD is a highly heterogeneous disorder resulting from gene-environment interactions. Even in patients with similar limitations in airflow, the clinical, physiologic and radiologic presentation of COPD can vary significantly from patient to patient. Therefore, to improve the management of COPD, it is important to identify clinically significant subgroups of COPD or "COPD phenotypes". ${ }^{3}$ A fraction of patients with COPD has inherited PiZZ (Glu342Lys) alpha-1 antitrypsin deficiency (A1ATD), a major genetic determinant influencing the development of early-onset COPD with emphysema, especially in cigarette smokers. ${ }^{4}$ The identification of biomarkers that can distinguish between A1ATD-PiZZ and non-A1ATD-PiMM COPD phenotypes could improve our understanding of the disease-driving mechanisms.

The use of proteomics is a growing trend in the search for new biomarkers of COPD. The advantage of this method is that it prompts various hypothesis-generating 
models, but not necessarily hypothesis-driven results. Current proteomic screenings in patients with COPD have involved mostly samples from the respiratory system, including bronchoalveolar lavage fluid, lung tissue, epithelial lining fluid and induced sputum. Several studies have investigated potential protein biomarkers in plasma and serum, ${ }^{5}$ and proteomic analyses have implicated proteins involved in the regulation of inflammation, oxidative stress, immune responses and structural proteins. ${ }^{6-9}$

Urine is one of the frequently used biofluids in clinical studies because it is easy to obtain in large quantities and it contains proteins and peptides, which are quite stable and less complex than in plasma. ${ }^{10}$ For these reasons, urine is an excellent reservoir of biomarkers (peptides, proteins and metabolites) for many diseases. ${ }^{11}$ For example, the levels of elastin degradation products, such as desmosine/ isodesmosine, have been investigated in the urine of COPD patients with and without A1ATD. ${ }^{12}$ However, urinary desmosine levels are variable and raise during exacerbations of COPD, limiting the application of this measurement in a clinical setting. ${ }^{13,14}$

A novel approach for identifying urinary biomarkers employs capillary electrophoresis (CE) coupled to mass spectrometry (MS). Previously, this method was rarely used, owing to the limitations associated with coupling CE to the MS instrument and the restricted loading capacity of CE capillaries. However, the significantly increased sensitivity of modern MS and optimized methods for coupling CE to MS have transformed CE-MS into an appropriate tool for profiling disease-associated metabolites in body fluid samples such as urine. CE-MS enables simultaneous quantification of hundreds of peptides in a urine sample, which can then be incorporated into a diagnostic or prognostic score. ${ }^{15}$ This method has enabled the identification of urinary biomarker classifiers that are highly specific for the diagnosis of various human diseases. ${ }^{10,16,17}$ Therefore, we hypothesized that urinary peptidome analysis using CE-MS may help to identify specific peptide profiles in PiZZ and PiMM patients with COPD.

\section{Materials and methods}

\section{Patients}

Nineteen clinically stable patients with COPD (10 females and 9 males; 16 ex-smokers and 3 never-smokers) were enrolled for urine analysis at the National Institute of Tuberculosis and Lung Diseases in Warsaw and at the Regional Centre of Pulmonology in Bydgoszcz. The Ethics Committee at the National Institute of Tuberculosis and Lung Diseases in Warsaw approved the study (KB-170/2011), and all subjects signed an informed consent form. Patients were subdivided according to their A1AT genotype: 7 with PiMM and 12 with PiZZ. Pulmonary function tests were performed according to the American Thoracic Society/European Respiratory Society criteria. Plasma levels of A1AT and other variables were analyzed at the clinical center in Warsaw.

\section{Urine samples}

Midstream morning urine was collected in sterile containers and stored at $-80^{\circ} \mathrm{C}$ prior to analysis, as recommended by the European Kidney and Urine Proteomics and Human Kidney and Urine Proteome Project and described previously. ${ }^{18}$ For analysis, urine was diluted in a solution containing $2 \mathrm{M}$ urea, $10 \mathrm{mM} \mathrm{NH}_{4} \mathrm{OH}$ and $0.02 \%(\mathrm{w} / \mathrm{v})$ sodium dodecyl sulfate. To remove higher molecular mass proteins, such as albumin and immunoglobulin $\mathrm{G}$, each sample was ultrafiltered using a Centrisart ultracentrifugation filter device $(20 \mathrm{kDa}$ cutoff Vivaspin; Sartorius, Goettingen, Germany) at 3,000× $g$ until $1.1 \mathrm{~mL}$ of filtrate was obtained. To remove urea, electrolytes and salts and to enrich polypeptides, the filtrate was then applied to a PD-10 desalting column (GE Healthcare BioSciences AB, Uppsala, Sweden) equilibrated with $0.01 \%$ $\mathrm{NH}_{4} \mathrm{OH}$ in high-performance liquid chromatography grade water (Roth, Bavaria, Germany). Finally, the samples were lyophilized and stored at $4^{\circ} \mathrm{C}$. Shortly before CE-MS analyses, the samples were dissolved in high-performance liquid chromatography grade water, as described elsewhere. ${ }^{19}$

\section{CE-MS analyses}

For analysis, the sample resuspension volume was adjusted to yield $0.8 \mu \mathrm{g} / \mu \mathrm{L}$ protein as measured by the BCA assay (Pierce Biotechnology, Rockford, IL, USA). CE-MS analyses were performed using a CE system (Beckman Coulter, Fullerton, CA, USA) coupled to a micrOTOF II MS (Bruker Daltonic, Bremen, Germany) by Mosaiques Diagnostics GmbH (Hannover, Germany), and the analyses passed all quality control criteria. Validation experiments were performed, and the reference signals of 1770 urinary polypeptides were used for CE-MS calibration. ${ }^{20}$ For normalization of the dilution variances, signal intensities were normalized to 29 housekeeping peptides. ${ }^{21}$ The obtained peaks indicated the molecular mass of each polypetide, the normalized $\mathrm{CE}$ migration (minutes) and the normalized signal intensities. Accuracy, precision, selectivity, sensitivity, reproducibility and stability of the CE-MS measurements were demonstrated elsewhere. ${ }^{22}$ All detected peptides were deposited, matched and annotated in a Microsoft SQL database to enable further statistical analyses. ${ }^{21}$ STATISTICA 8.0 (StatSoft Inc., Tulsa, 
OK, USA) was used for the data calculations. Functional analysis was performed using Gene Group Functional Profiling, g:Profiler (http://biit.cs.ut.ee/gprofiler/). ${ }^{23}$

\section{Results}

\section{Patient characteristics}

General patient characteristics are summarized in Table 1. Our cohort included patients having different severity of COPD: five PiZZ cases and one PiMM case were categorized as very severe (forced expiratory volume in 1 second $\left[\mathrm{FEV}_{1}\right]<35 \%$ ), seven PiZZ and five PiMM cases as severe

Table I Characteristics of COPD patients

\begin{tabular}{|c|c|c|c|c|}
\hline \multirow[t]{2}{*}{ Variables } & \multicolumn{2}{|c|}{ AIAT genotype } & \multicolumn{2}{|c|}{ Mann-Whitney test } \\
\hline & $\begin{array}{l}P i Z Z \\
(n=12)\end{array}$ & $\begin{array}{l}\text { PiMM } \\
(n=7)\end{array}$ & $P$-value & Z-adjusted \\
\hline Age (years) & $54.7(9)$ & $65.7(6.3)$ & $1.21 \mathrm{E}-02$ & -2.51 \\
\hline Gender, M/F & $5 / 7$ & $4 / 3$ & & \\
\hline $\begin{array}{l}\text { Smoking history, } \\
\text { never/ex-smokers }\end{array}$ & $1 / 11$ & $2 / 5$ & & \\
\hline Pack-years & $19.2(\mathrm{II})$ & $32.9(26)$ & ns & -1.84 \\
\hline $\mathrm{FEV}_{1} \%$ & $37.6(13.8)$ & $43.7(11.2)$ & ns & -0.63 \\
\hline $\mathrm{FEV}_{1}(\mathrm{I})$ & I.I (0.4) & I.I (0.6) & ns & 0.08 \\
\hline $\mathrm{FEV}, \% / \mathrm{FVC}$ & $37(10.2)$ & $47.3(12.5)$ & ns & -1.14 \\
\hline FVC (I) & $3(0.9)$ & $2.5(1.2)$ & ns & 0.78 \\
\hline FVC\% & $78.1(15.8)$ & $77.5(12.7)$ & ns & 0.21 \\
\hline VC (I) & $3.2(0.8)$ & $1.4(0.3)$ & 4.I3E-02 & 2.04 \\
\hline VC\% & $84.8(14.8)$ & $75.4(16.1)$ & ns & 0.75 \\
\hline PEF (I) & $3.1(0.8)$ & $1.8(0.3)$ & ns & 1.83 \\
\hline PEF\% & $4 I .8(I I .4)$ & $38.5(6.5)$ & ns & 0.43 \\
\hline AIAT (mg/dL) & $22.5(5.4)$ & $166.3(22.9)$ & $4.53 \mathrm{E}-04$ & -3.51 \\
\hline hsCRP (mg/dL) & $0.8(1.4)$ & $0.9(0.7)$ & ns & -0.47 \\
\hline ALT (U/L) & $28.8(14.9)$ & $17.6(3.3)$ & ns & 1.77 \\
\hline AST (U/L) & $26.5(9)$ & $19(3)$ & ns & 0.94 \\
\hline Leukocytosis (10\%/L) & $9.5(3.5)$ & $8.1(1.5)$ & ns & 0.49 \\
\hline Neutrophils (10/L) & $6.1(2.7)$ & $5.6(2.1)$ & ns & 0.14 \\
\hline Neutrophils (\%) & $63(6.4)$ & $60.8(5.2)$ & ns & 0.64 \\
\hline Lymphocytes (10/L) & $2.3(0.5)$ & $2.5(0.6)$ & ns & -0.49 \\
\hline Lymphocytes \% & $26.2(7.8)$ & $28.3(3.5)$ & ns & -0.21 \\
\hline Monocytes (10\%/L) & $0.8(0.5)$ & $0.8(0.1)$ & ns & -1.34 \\
\hline Monocytes \% & $8.1(2.5)$ & $9.6(1.1)$ & ns & -0.78 \\
\hline Eosinophils (10/L) & $0.3(0.4)$ & $0.1(0.1)$ & ns & 1.01 \\
\hline Eosinophils \% & $2.8(3.3)$ & I.I (0.8) & ns & 0.93 \\
\hline $\mathrm{PO}_{2}$ (Torr) & $60.3(8.5)$ & $52.9(11.4)$ & ns & 0.85 \\
\hline $\mathrm{pCO}_{2}$ (Torr) & $38.2(7)$ & $43.8(6.3)$ & ns & -0.76 \\
\hline $\mathrm{SO}_{2} \%$ & $91.6(3.5)$ & $86.3(5.4)$ & ns & 1.04 \\
\hline $\mathrm{pH}$ & $7.5(0.03)$ & $7.4(0.03)$ & ns & 1.43 \\
\hline $\mathrm{HCO}_{3}^{-}(\mathrm{mmol} / \mathrm{L})$ & $25.6(3.7)$ & $27.1(2.8)$ & ns & -0.66 \\
\hline $\mathrm{BE}(\mathrm{mmol} / \mathrm{L})$ & $1.7(3.4)$ & $2.6(2.6)$ & ns & -0.66 \\
\hline
\end{tabular}

Note: All data are presented as mean (standard deviation).

Abbreviations: AIAT, $\alpha \mathrm{I}$-antitrypsin; ALT, alanine transaminase; AST, aspartate transaminase; $\mathrm{BE}$, base excess/deficit; ECM, extracellular matrix; $\mathrm{FEV}_{1}$, forced expiratory volume in I second; $\mathrm{FVC}$, forced vital capacity; $\mathrm{HCO}_{3}$, hydrogen carbonate; hsCRP, high-sensitive C-reactive protein; $\mathrm{PCO}_{2}$, partial pressure of carbon dioxide; PEF, peak expiratory flow; $\mathrm{pO}_{2}$, partial pressure of oxygen; $\mathrm{SO}_{2}$, oxygen saturation; VC, vital capacity. $\left(35 \%<\mathrm{FEV}_{1}<49 \%\right)$ and one PiMM case as moderate $\left(50 \%<\mathrm{FEV}_{1}<79 \%\right)$. As expected, the patients with PiZZ were younger than those with PiMM (mean [standard deviation $\{\mathrm{SD}\}$ ]: $54.67 \pm 9.03$ vs $65.71 \pm 6.27$ years, respectively, $P<0.05$ ) and had lower plasma levels of A1AT (mean [SD]: PiZZ 22.52 $\pm 5.43 \mathrm{mg} / \mathrm{dL}$ vs PiMM $166.29 \pm 22.89 \mathrm{mg} / \mathrm{dL}$, $P<0.0001)$. The patient groups did not differ statistically in terms of the pulmonary function tests, anthropometric measures and other serum parameters (Table 1).

\section{Functional clustering analysis}

We identified over 4200 peptides in each urine sample; however, only 66 peptides (corresponding to 36 unique proteins) differed between the PiMM and PiZZ COPD cases. We used the web server g:Profiler to predict the function of 36 proteins (http://biit.cs.ut.ee/gprofiler/). ${ }^{23}$ Functional analysis revealed that the differentially expressed peptides were clustered in significant gene ontology categories related to biologic processes, cellular components, molecular functions and biologic pathways. As shown in Table 2, most of the peptides are related to the regulation of immune processes, collagen catabolism and biosynthesis, endoplasmic reticulum, protein digestion, the PI3K-Akt pathway and scavenging by class A receptor, among others.

\section{Urinary peptides distinguish $\mathrm{PiZZ}$ and PiMM COPD cases}

Urinary peptides from the collagen family were the most abundant and divergent in our patient cohort. Specifically, COL4A5, COL5A3, COL7A1, COL8A2, COL9A3, COL11A1 and COL23A1 were more frequent in the COPD cases with PiMM, whereas COL4A1 and COL4A3 were more frequent in the COPD cases with PiZZ. Further analysis revealed that COL1A1 (number 15 , Table $3 ; 100 \%$ sensitivity, $85.71 \%$ specificity, $P<0.005)$ and both COL5A3 and COL3A1 (numbers 52 and 62, Table 3; $91.67 \%$ sensitivity, $85.71 \%$ specificity, $P<0.005$ ) were useful for discriminating between the PiZZ and PiMM groups.

Interestingly, cases with PiZZ COPD presented low levels of urinary peptides involved in lipoproteins/lipids and retinoic acid metabolism, such as apolipoprotein A-I and $\mathrm{C} 4$, retinol-binding protein 4 and prostaglandin- $\mathrm{H} 2$ D-isomerase. In particular, peptides of apolipoprotein $\mathrm{C} 4$ (number 60, Table 3) discriminated PiZZ from PiMM cases with a sensitivity of $91.67 \%$ and a specificity of $85.71 \%$ $(P<0.005)$. However, peptides of MDS1 and EVII complex locus, gelsolin and hemoglobin alpha, were found in the urine of PiZZ, but not PiMM cases (Table 3). We identified two 
Table 2 The 36 proteins functionally clustered by g:Profiler

\begin{tabular}{|c|c|c|c|}
\hline Biologic process & Count & $P$-value & Genes \\
\hline Collagen catabolic process & 11 & $4 e-16$ & $\begin{array}{l}\text { COLIIAI, COL5A3, COLIAI, COL7AI, COL2AI, COLIA2, } \\
\text { COL3AI, COL4A3, COL8A2, COL4AI, COL4A5 }\end{array}$ \\
\hline ECM organization & 13 & $2 \mathrm{e}-1 \mathrm{I}$ & $\begin{array}{l}\text { COLIIAII, COL5A3, COL9A3, COLIAI, COL7AI, COL2AI, GSN, } \\
\text { COLIA2, COL3AI, COL4A3, COL8A2, COL4AI, COL4A5 }\end{array}$ \\
\hline Collagen fibril organization & 6 & le-07 & COLIIAI, COL5A3, COLIAI, COL2AI, COLIA2, COL3AI \\
\hline Anatomic structure morphogenesis & 17 & $4 \mathrm{e}-04$ & $\begin{array}{l}\text { COLIIAI, COLIAI, COL7AI, APOAI, ANXAI, RBP4, COL2AI, } \\
\text { GSN, RUNXI, MYL3, COLIA2, COL3AI, COL4A3, KRTI3, } \\
\text { COL8A2, BCL9L, COL4AI }\end{array}$ \\
\hline $\begin{array}{l}\text { Collagen-activated tyrosine kinase } \\
\text { receptor signaling pathway }\end{array}$ & 3 & $5 e-04$ & COL4A3, COL4AI, COL4A5 \\
\hline Animal organ development & 19 & $5 e-04$ & $\begin{array}{l}\text { COLIIAI, COL5A3, COL9A3, COLIAI, APOAI, ANXAI, RBP4, } \\
\text { COL2AI, RUNXI, MYL3, COLIA2, B2M, COL3AI, COL4A3, } \\
\text { KRTI3, COL8A2, BCL9L, KRTIO, COL4AI }\end{array}$ \\
\hline Circulatory system development & 11 & le-03 & $\begin{array}{l}\text { COLIIAI, COLIAI, RBP4, COL2AI, RUNXI, MYL3, COLIA2, } \\
\text { COL3AI, COL4A3, COL8A2, COL4AI }\end{array}$ \\
\hline Cellular response to amino acid stimulus & 4 & $8 e-03$ & COLIAI, COLIA2, COL3AI, COL4AI \\
\hline Sensory perception of sound & 5 & le-02 & COLIIAI, COLIAI, COL2AI, COL4A3, ESPN \\
\hline Tissue development & 13 & $2 e-02$ & $\begin{array}{l}\text { COLIIAI, COLIAI, COL7AI, ANXAI, RBP4, COL2AI, GSN, } \\
\text { RUNXI, MYL3, COL3AI, BCL9L, KRTIO, COL4AI }\end{array}$ \\
\hline Receptor-mediated endocytosis & 6 & $2 \mathrm{e}-02$ & UNCII9, APOAI, SNX9, B2M, HBAI, HBB \\
\hline Response to wounding & 8 & $4 e-02$ & $\begin{array}{l}\text { COLIAI, APOAI, ANXAI, GSN, COLIA2, COL3AI, SERPINAI, } \\
\text { HBB }\end{array}$ \\
\hline Regulation of immune system process & 11 & $5 e-02$ & $\begin{array}{l}\text { COLIAI, APOAI, IRAK2, ANXAI, RBP4, COL2AI, GSN, RUNXI, } \\
\text { COLIA2, B2M, COL3AI }\end{array}$ \\
\hline \multicolumn{4}{|l|}{ Cellular component } \\
\hline Endoplasmic reticulum lumen & 16 & $9 e-20$ & $\begin{array}{l}\text { COL23AI, COLIIAI, COL5A3, COL9A3, COLIAI, COL7AI, } \\
\text { APOAI, COL2AI, COLIA2, B2M, COL3AI, COL4A3, COL8A2, } \\
\text { COL4AI, COL4A5, SERPINAI }\end{array}$ \\
\hline Complex of collagen trimers & 10 & $3 e-19$ & $\begin{array}{l}\text { COLIIAI, COL5A3, COLIAI, COL7AI, COL2AI, COLIA2, } \\
\text { COL3AI, COL4A3, COL4AI, COL4A5 }\end{array}$ \\
\hline Endocytic vesicle lumen & 3 & $9 e-03$ & $A P O A I, H B A I, H B B$ \\
\hline Vesicle & 19 & $2 \mathrm{e}-02$ & $\begin{array}{l}\text { COL5A3, CHGB, PTGDS, COLIAI, COL7AI, APOAI, SNX9, } \\
\text { IRAK2, ANXAI, FXYD2, RBP4, GSN, COLIA2, B2M, KRTI3, } \\
\text { KRTIO, SERPINAI, HBAI, HBB }\end{array}$ \\
\hline \multicolumn{4}{|l|}{ Molecular function } \\
\hline ECM structural constituent & 11 & $2 \mathrm{e}-15$ & $\begin{array}{l}\text { COLIIAI, COL5A3, COL9A3, COLIAI, COL2AI, COLIA2, } \\
\text { COL3AI, COL4A3, COL8A2, COL4AI, COL4A5 }\end{array}$ \\
\hline Platelet-derived growth factor binding & 5 & $2 e-08$ & COLIAI, COL2AI, COLIA2, COL3AI, COL4AI \\
\hline Haptoglobin binding & 2 & $2 e-02$ & $H B A I, H B B$ \\
\hline \multicolumn{4}{|l|}{ Biologic pathway (KEGG) } \\
\hline Protein digestion and absorption & 12 & $5 e-16$ & $\begin{array}{l}\text { COLIIAI, COL5A3, COL9A3, COLIAI, COL7AI, FXYD2, } \\
\text { COL2AI, COLIA2, COL3AI, COL4A3, COL4AI, COL4A5 }\end{array}$ \\
\hline ECM-receptor interaction & 7 & $2 \mathrm{e}-07$ & COL9A3, COLIAI, COL2AI, COLIA2, COL4A3, COL4AI, COL4A5 \\
\hline Amebiasis & 6 & $3 e-05$ & COLIAI, COLIA2, COL3AI, COL4A3, COL4AI, COL4A5 \\
\hline $\begin{array}{l}\text { AGE-RAGE signaling pathway in } \\
\text { diabetic complications }\end{array}$ & 6 & $3 e-05$ & COLIAI, COLIA2, COL3AI, COL4A3, COL4AI, COL4A5 \\
\hline Focal adhesion & 7 & le-04 & COL9A3, COLIAI, COL2AI, COLIA2, COL4A3, COL4AI, COL4A5 \\
\hline PI3K/Akt signaling pathway & 7 & $4 e-03$ & COL9A3, COLIAI, COL2AI, COLIA2, COL4A3, COL4AI, COL4A5 \\
\hline African trypanosomiasis & 3 & $8 e-03$ & $A P O A I, H B A I, H B B$ \\
\hline \multicolumn{4}{|l|}{ Biologic pathway (reactome) } \\
\hline $\begin{array}{l}\text { Collagen biosynthesis and modifying } \\
\text { enzymes }\end{array}$ & 13 & $7 e-19$ & $\begin{array}{l}\text { COL23AI, COLIIAI, COL5A3, COL9A3, COLIAI, COL7AI, } \\
\text { COL2AI, COLIA2, COL3AI, COL4A3, COL8A2, COL4AI, COL4A5 }\end{array}$ \\
\hline Scavenging by Class $\mathrm{A}$ receptors & 5 & $6 e-07$ & COLIAI, APOAI, COLIA2, COL3AI, COL4AI \\
\hline NCAMI interactions & 4 & $\mathrm{Ie}-03$ & COL9A3, COL4A3, COL4AI, COL4A5 \\
\hline
\end{tabular}

Abbreviations: RAGE, receptor for advanced glycation endproduct; AGE, advanced glycation endproduct; NCAMI, neural cell adhesion molecule I; KEGG, Kyoto Encyclopedia of Genes and Genomes; ECM, extracellular matrix. 
Table 3 Urinary peptides distinguish PiZZ and PiMM COPD cases

\begin{tabular}{|c|c|c|c|c|c|c|c|c|}
\hline \multirow[t]{2}{*}{ Pep } & \multirow[t]{2}{*}{ Protein name } & \multirow{2}{*}{$\begin{array}{l}\text { UniProt } \\
\text { AC }\end{array}$} & \multirow{2}{*}{$\begin{array}{l}\text { Exp } \\
\text { Mass }\end{array}$} & \multirow[t]{2}{*}{ Peptide sequence } & \multicolumn{2}{|c|}{ Intensity, mean (SD) } & \multirow[t]{2}{*}{ Z-adjusted } & \multirow[t]{2}{*}{ FDR } \\
\hline & & & & & $\overline{P i Z Z}$ & PiMM & & \\
\hline I & Hemoglobin subunit $\beta$ & P6887I & 2,659 & FESFGDLSTPDAVMGNPKVKAHGKK & $148(191)$ & $519(379)$ & $2.1 *$ & $2 \mathrm{E}-03$ \\
\hline 2 & $\beta$-2-Microglobulin & P6I769 & ।,077 & IVKWDRDM & $16(37)$ & $127(12 \mid)$ & $2.9 * *$ & $2 \mathrm{E}-03$ \\
\hline 3 & $\begin{array}{l}\text { MDSI and EVII complex locus } \\
\text { protein MDSI }\end{array}$ & QI3465 & 1,140 & TSHSSSNVWH & $3,724(4,315)$ & $0(0)$ & $-2.6 * *$ & $3 \mathrm{E}-03$ \\
\hline 4 & Collagen $\alpha-I(I)$ chain & P02452 & 1,900 & SPGRDGSPGAKGDRGETGPA & $12(4 \mid)$ & $259(26 I)$ & $2.5^{*}$ & IE-03 \\
\hline 5 & Collagen $\alpha-I(I I I)$ chain & P0246I & $\mathrm{I}, 354$ & KGEPGGPGADGVPGK & $592(389)$ & $\mathrm{I}, 100(55 \mathrm{I})$ & $2^{*}$ & $8 \mathrm{E}-03$ \\
\hline 6 & Collagen $\alpha-I(I I I)$ chain & P0246I & 2,154 & NGEPGGKGERGAPGEKGEGGPPG & $92(142)$ & $205(82)$ & $2 . I^{*}$ & $3 \mathrm{E}-03$ \\
\hline 7 & Retinol-binding protein 4 & P02753 & 1,439 & LQKGNDDHWIVD & $242(623)$ & $390(243)$ & $2.2 *$ & $5 \mathrm{E}-03$ \\
\hline 8 & Collagen $\alpha-I(I)$ chain & P02452 & 1,355 & GQPGAKGEPGDAGAK & $46(43)$ & $108(69)$ & $2 *$ & $8 \mathrm{E}-03$ \\
\hline 9 & Collagen $\alpha-I(I)$ chain & P02452 & I,877 & DDGEAGKPGRPGERGPPGP & $1,985(715)$ & $3,634(1,405)$ & $2 . I^{*}$ & $3 \mathrm{E}-03$ \\
\hline 10 & Gelsolin & P06396 & 2,232 & DEELGGTPVQSRVVQGKEPAH & $120(176)$ & $0(0)$ & $-2.2^{*}$ & $2 \mathrm{E}-03$ \\
\hline 11 & Hemoglobin subunit $\alpha$ & P69905 & 2,021 & AAHLPAEFTPAVHASLDKF & $21(25)$ & $0(0)$ & $-2.2^{*}$ & $3 \mathrm{E}-03$ \\
\hline 12 & Keratin; type I cytoskeletal I3 & $\mathrm{PI} 3646$ & $\mathrm{I}, 36 \mathrm{I}$ & EKITMQNLNDR & $214(464)$ & $389(246)$ & $2^{*}$ & $9 \mathrm{E}-03$ \\
\hline 13 & Apolipoprotein A-I & P02647 & $\mathrm{I}, 524$ & SALEEYTKKLNTQ & $0(0)$ & $179(425)$ & $2.4^{*}$ & $2 \mathrm{E}-03$ \\
\hline 14 & $\lg \lambda-2$ chain $C$ regions & P0CG05 & 3,202 & $\begin{array}{l}\text { WKADSSPVKAGVETTTPS } \\
\text { KQSNNKYAASSY }\end{array}$ & $44(125)$ & $98(66)$ & $2.5^{*}$ & $6 \mathrm{E}-04$ \\
\hline 15 & Collagen $\alpha-I(I)$ chain & P02452 & 858 & SPGEAGRPG & $410(227)$ & $134(130)$ & $-3 * *$ & $\mathrm{IE}-02$ \\
\hline 16 & Collagen $\alpha-I(V I I)$ chain & Q02388 & 2,410 & GLKGDRGDPGPQGPPGLALGERGPP & $60(200)$ & III (II3) & $2^{*}$ & $3 \mathrm{E}-03$ \\
\hline 17 & Prostaglandin-H2 D-isomerase & $\mathrm{P} 4 \mid 222$ & 1,843 & YSQGSKGPGEDFRMATL & $20(40)$ & $61(63)$ & $2.2 *$ & $3 \mathrm{E}-03$ \\
\hline 18 & Annexin $\mathrm{Al}$ & P04083 & 2,057 & FIENEEQEYVQTVKSSK & $0(0)$ & $34(62)$ & $2.4^{*}$ & $\mathrm{IE}-03$ \\
\hline 19 & $\alpha$-I-antitrypsin & P01009 & 1,943 & EAIPMSIPPEVKFNKPFV & $0(0)$ & $16,167(39,363)$ & $2.4^{*}$ & IE-03 \\
\hline 20 & Myosin light chain 3 & P08590 & 1,739 & PAPAPPPEPERPKEVE & $362(216)$ & $587(265)$ & $2^{*}$ & $4 \mathrm{E}-03$ \\
\hline 21 & $\alpha-I$-antitrypsin & P01009 & 2,042 & EAIPMSIPPEVKFNKPFV & $0(0)$ & $9,238(22,506)$ & $2.4^{*}$ & $\mathrm{IE}-03$ \\
\hline 22 & Collagen $\alpha-I(I I)$ chain & P02458 & 2,133 & GARGPEGAQGPRGEPGTPGSPGP & $\mathrm{I}, \mathrm{I} 52(687)$ & $2,266(1,111)$ & $2 . I^{*}$ & $3 \mathrm{E}-03$ \\
\hline 23 & Collagen $\alpha-2(I)$ chain & P08I23 & 1,226 & GPPGPDGNKGEPG & $539(73)$ & $690(176)$ & $2.2 *$ & $8 \mathrm{E}-03$ \\
\hline 24 & Collagen $\alpha-I(I)$ chain & P02452 & $|, 2| \mathrm{I}$ & SPGPDGKTGPPGP & $0(0)$ & $94(179)$ & $2.4^{*}$ & $5 \mathrm{E}-03$ \\
\hline 25 & Collagen $\alpha-I(I)$ chain & P02452 & $\mathrm{I}, 408$ & GPPGEAGKPGEQGVP & $149(287)$ & $353(339)$ & $2.1 *$ & $7 \mathrm{E}-03$ \\
\hline 26 & Hemoglobin subunit $\beta$ & P6887I & $2,|7|$ & TPEEKSAVTALWGK VNVDEV & $0(0)$ & $34 I(469)$ & $2.8^{* *}$ & $3 \mathrm{E}-04$ \\
\hline 27 & Collagen $\alpha-I(I)$ chain & P02452 & 2,150 & DGQPGAKGEPGDAGAKGDAGPPGP & $\mathrm{I}, 774(9 \mid 3)$ & $3,022(1,085)$ & $2.6 * *$ & 7E-04 \\
\hline 28 & Collagen $\alpha-2(I)$ chain & P08I23 & $1,17 \mid$ & DQGPVGRTGEVG & $54(113)$ & $0(0)$ & $-2.2^{*}$ & IE-02 \\
\hline 29 & Collagen $\alpha-I(I I I)$ chain & P0246I & 1,423 & GLPGTGGPPGENGKPG & $2,34 \mid(I, 4 \mid 0)$ & $3,863(1,416)$ & $2^{*}$ & $7 \mathrm{E}-03$ \\
\hline \multirow[t]{2}{*}{30} & Collagen $\alpha-I(I)$ chain & P02452 & 2,584 & AGPPGADGQPGAKGEPG & $4(13)$ & $4 I(6 I)$ & $2.2^{*}$ & $2 \mathrm{E}-03$ \\
\hline & & & & DAGAKGDAGPPGP & & & & \\
\hline 31 & Collagen $\alpha-I(I I)$ chain & P02458 & 1,096 & APGEDGRPGPP & $0(0)$ & $12(18)$ & $2.4^{*}$ & $8 \mathrm{E}-03$ \\
\hline 32 & $\begin{array}{l}\text { Sodium/potassium- } \\
\text { transporting ATPase subunit } \gamma\end{array}$ & P547I0 & $|, 43|$ & LSMDGGGSPKGDVDP & $56(90)$ & $169(138)$ & $2^{*}$ & $8 \mathrm{E}-03$ \\
\hline 33 & Collagen $\alpha-I(I I I)$ chain & P0246I & 1,660 & GPPGPPGTSGHPGSPGSPG & $202(228)$ & $714(282)$ & $3 * *$ & $3 \mathrm{E}-04$ \\
\hline 34 & Collagen $\alpha-3(\mathrm{IV})$ chain & Q01955 & 1,628 & PGPPGPPGPPGHPGPQGP & $205(124)$ & $71(95)$ & $-2^{*}$ & $6 \mathrm{E}-03$ \\
\hline 35 & Collagen $\alpha-I(\mathrm{IV})$ chain & P02462 & ।,7। & GPPGPPGPPGPPGEKGQM & $127(4 \mid 2)$ & $50(47)$ & $2.1^{*}$ & $4 \mathrm{E}-03$ \\
\hline 36 & Collagen $\alpha-I(X X \mid I I)$ chain & Q86Y22 & $\mathrm{I}, 60 \mathrm{I}$ & DPGPPGQSGRDGYPGP & $49(60)$ & $189(104)$ & $2.3^{*}$ & $3 \mathrm{E}-03$ \\
\hline \multirow[t]{2}{*}{37} & Collagen $\alpha-3(I X)$ chain & Q14050 & 3,048 & QGDRGDKGAAGAGLDG & $33(67)$ & $169(146)$ & $2.3^{*}$ & $\mathrm{IE}-03$ \\
\hline & & & & PEGDQGPQGPQGVPGTS & & & & \\
\hline 38 & $\begin{array}{l}\text { Sodium/potassium- } \\
\text { transporting ATPase subunit } \gamma\end{array}$ & P547I0 & $\mathrm{I}, 504$ & GLSMDGGGSPKGDVDP & $0(0)$ & $42(49)$ & $2.4^{*}$ & $2 \mathrm{E}-03$ \\
\hline 39 & Collagen $\alpha-5(\mathrm{IV})$ chain & P29400 & 1,627 & PGAPGFPGSKGEPGDIL & 101 (I77) & $460(502)$ & $2^{*}$ & $5 \mathrm{E}-03$ \\
\hline 40 & Collagen $\alpha-I(X I)$ chain & $\mathrm{PI} 2107$ & $\mathrm{I}, 734$ & PPGPKGNMGPQGEPGPPG & $0(0)$ & $62(69)$ & $3.3^{* *}$ & IE-04 \\
\hline \multirow[t]{2}{*}{41} & Collagen $\alpha-I(I)$ chain & P02452 & 2,946 & NSGEPGAPGSKGDTGAKG & $4(8)$ & $31(34)$ & $2.2^{*}$ & $2 \mathrm{E}-03$ \\
\hline & & & & EPGPVGVQGPPGPAG & & & & \\
\hline 42 & Collagen $\alpha-I(X I)$ chain & $\mathrm{PI} 2107$ & $\mathrm{I}, 675$ & KGENGDVGPMGPPGPPGP & $28(39)$ & $215(185)$ & $2 . I^{*}$ & $4 \mathrm{E}-03$ \\
\hline 43 & Collagen $\alpha-2(\mathrm{VIII})$ chain & P25067 & 1,822 & GPPGEGRAGEPGTAGPTGPP & $314(378)$ & $848(304)$ & $2.5^{*}$ & $\mathrm{IE}-03$ \\
\hline 44 & Collagen $\alpha-I$ chain & P0246I & 1,652 & QPGEKGSPGAQGPPGAPG & $4(10)$ & $79(57)$ & $3.1 * *$ & $2 \mathrm{E}-04$ \\
\hline 45 & Prostaglandin-H2 D-isomerase & P4I222 & 1,805 & AQVSVQPNFQQDKFLG & $0(0)$ & $32(46)$ & $2.4^{*}$ & $2 \mathrm{E}-03$ \\
\hline \multirow[t]{2}{*}{46} & Secretogranin-I & P05060 & 3,202 & SSQGGSLPSEEKGHPQEES & $43(68)$ & $220(192)$ & $2.3^{*}$ & $\mathrm{IE}-03$ \\
\hline & & & & EESNVSMASLGE & & & & \\
\hline
\end{tabular}


Table 3 (Continued)

\begin{tabular}{|c|c|c|c|c|c|c|c|c|}
\hline \multirow[t]{2}{*}{ Pep } & \multirow[t]{2}{*}{ Protein name } & \multirow{2}{*}{$\begin{array}{l}\text { UniProt } \\
\text { AC }\end{array}$} & \multirow{2}{*}{$\begin{array}{l}\text { Exp } \\
\text { Mass }\end{array}$} & \multirow{2}{*}{ Peptide sequence } & \multicolumn{2}{|c|}{ Intensity, mean (SD) } & \multirow[t]{2}{*}{ Z-adjusted } & \multirow[t]{2}{*}{ FDR } \\
\hline & & & & & PiZZ & PiMM & & \\
\hline 47 & Collagen $\alpha-2(I)$ chain & P08I23 & $\mathrm{I}, 853$ & NGAPGEAGRDGNPGNDGPPG & II 8 (128) & $13(26)$ & $-2.2 *$ & $2 \mathrm{E}-03$ \\
\hline 48 & Collagen $\alpha-I(I I I)$ chain & P0246I & $\mathrm{I}, 860$ & NPGPPGPSGSPGKDGPPGPAG & $282(201)$ & $81(101)$ & $-2.5^{*}$ & IE-03 \\
\hline \multirow[t]{2}{*}{49} & Collagen $\alpha-I(I I)$ chain & P02458 & 3,458 & TGPPGPAGFAGPPGADGQP & $23,598(6,494)$ & $11,9 \mid 5(9,047)$ & $-2.3 *$ & IE-03 \\
\hline & & & & GAKGEQGEAGQKGDAGAPGP & & & & \\
\hline 50 & Collagen $\alpha-I(I)$ chain & P02452 & 1,685 & EPGSPGENGAPGQMGPR & $0(0)$ & $395(953)$ & $2.4^{*}$ & $2 \mathrm{E}-03$ \\
\hline 51 & Collagen $\alpha-I(I)$ chain & P02452 & 1,826 & GANGAPGNDGAKGDAGAPGAPG & $493(4 \mid 4)$ & $130(126)$ & $-2 . I^{*}$ & $3 \mathrm{E}-03$ \\
\hline 52 & Collagen $\alpha-3(V)$ chain & P25940 & 1,699 & GIDGSPGEKGDPGDVGGPG & I (4) & $39(33)$ & $3.4 * * *$ & $8 \mathrm{E}-05$ \\
\hline 53 & Keratin; type I cytoskeletal I0 & PI3645 & I,737 & TQLLNNMRSQYEQL & $0(0)$ & $244(431)$ & $2.8 * *$ & $5 \mathrm{E}-04$ \\
\hline 54 & Collagen $\alpha-(I)$ chain & P02452 & I,762 & QGPGGPPGPKGNSGEPGAPG & $0(0)$ & $34(57)$ & $2.4^{*}$ & $2 \mathrm{E}-03$ \\
\hline \multirow[t]{2}{*}{55} & Runt-related transcription & Q01196 & 3,443 & SISDPRMHYPGAFTYSP & $3(9)$ & $81(74)$ & $2.9^{* *}$ & $2 \mathrm{E}-04$ \\
\hline & factor I & & & TPVTSGIGIGMSAMGSA & & & & \\
\hline 56 & Collagen $\alpha-I(I)$ chain & P02452 & 2,104 & GPPGEAGKPGEQGVPGDLGAPGP & $\mathrm{I}, 63 \mathrm{I}(447)$ & $2,430(662)$ & $2.6 * *$ & $7 \mathrm{E}-04$ \\
\hline \multirow[t]{2}{*}{57} & Interleukin-I receptor- & 043187 & 2,192 & ANGSLQDRLQGQGGSDPLPWP & $2(5)$ & $15(15)$ & $2.1 *$ & $3 \mathrm{E}-03$ \\
\hline & associated kinase-like 2 & & & & & & & \\
\hline \multirow[t]{2}{*}{58} & Collagen $\alpha-2(I)$ chain & P08I23 & 3,633 & DQGPVGRTGEVGAVG & $10(32)$ & $132(105)$ & $3.2^{* *}$ & 7E-05 \\
\hline & & & & PPGFAGEKGPSGEAGTAGPPGTPGP & & & & \\
\hline \multirow[t]{2}{*}{59} & Sorting nexin-9 & Q9Y5XI & 2,501 & ASTAQASSSAASNNHQ & $19(28)$ & $107(104)$ & $2.2^{*}$ & $2 \mathrm{E}-03$ \\
\hline & & & & VGSGNDPWSA & & & & \\
\hline 60 & Apolip oprotein C-IV & P55056 & 2,418 & TQQPQQDEMPSPTFLTQVKES & $8(20)$ & $67(32)$ & $3.3^{* * * *}$ & $6 \mathrm{E}-05$ \\
\hline \multirow[t]{2}{*}{61} & Collagen $\alpha-5(\mathrm{IV})$ chain & P29400 & 2,646 & GQDGIPGPAGQKGEPG & $0(0)$ & $25(47)$ & $2.4^{*}$ & IE-03 \\
\hline & & & & QPGFGNPGPPGL & & & & \\
\hline \multirow[t]{2}{*}{62} & Collagen $\alpha-I(I I I)$ chain & P0246I & 2,854 & PQGPPGPTGPGGDKGD & $2,570(2,224)$ & $8,157(6,403)$ & $2.5^{*}$ & 7E-04 \\
\hline & & & & TGPPGPQGLQGLPGT & & & & \\
\hline 63 & Espin & BIAK53 & $\mathrm{I}, 005$ & LPPPPPPPPP & $17(19)$ & $62(5 \mathrm{I})$ & $2 *$ & $3 \mathrm{E}-02$ \\
\hline 64 & Collagen $\alpha-I(I I)$ chain & P02458 & I,594 & PGTPGNPGPPGPPGPPGP & $110(187)$ & $8(21)$ & $-2.2 *$ & $4 \mathrm{E}-03$ \\
\hline 65 & Protein unc-II 9 homolog $A$ & Q13432 & $\mathrm{I}, 247$ & SESGSESEPDAGP & $2(6)$ & $23(35)$ & $2.2 *$ & $6 \mathrm{E}-03$ \\
\hline 66 & $\begin{array}{l}\text { B-cell CLL/lymphoma 9-like } \\
\text { protein }\end{array}$ & Q86UU0 & 1,557 & PGMGWTEDLPPMGGP & $888(43 I)$ & $443(255)$ & $-2 . I^{*}$ & $5 \mathrm{E}-03$ \\
\hline
\end{tabular}

Notes: Statistical results based on Mann-Whitney U-test and FDR test; Exp Mass (molecular mass of the peptide, kDa), the Z-adjusted reported the equivalent $P$-values as follows: $* P<0.05 ; * * P<0.0$ I; $* * * P>0.001$.

Abbreviations: CLL, chronic lymphocytic leukemia; FDR, false discovery rate; SD, standard deviation.

peptides of A1AT in the urine of $43 \%$ of PiMM cases, but they were absent in PiZZ cases.

\section{Discussion}

The increased proteolytic activity and protease-antiprotease imbalance in COPD have been well described. Therefore, many studies have focused on identifying protease-generated peptides in various biologic fluids from patients with COPD.${ }^{24}$ Recently, Wendt et al have used liquid chromatography coupled to MS for metabolomic profiling of bronchial lavage fluid obtained from patients with COPD and further confirmed a significant increase in peptides compared with healthy controls. ${ }^{25}$

PiZZ A1ATD-related COPD is associated with low circulating levels of A1AT (10\%-15\% of normal levels), which is the main inhibitor of neutrophil proteases. Neutrophils are among the key participants in COPD, and they secrete serine proteases (neutrophil elastase, cathepsin $\mathrm{G}$ and proteinase-3) and matrix metalloproteinase (MMP)- 8 and
MMP-9 ${ }^{26,27}$ Active elastase can degrade many extracellular matrix substances, including elastin, collagen types I-IV and fibronectin, and activate MMPs. ${ }^{28}$ For example, it has been demonstrated that elastase, cathepsin $\mathrm{G}$ and proteinase-3 activate MMP-2, and this activation is blocked by A1AT, but not by an MMP-inhibitor. ${ }^{29}$ Macrophages also secrete proteases, including MMP-2, MMP-9 and MMP-12, and cathepsins K, L and S. Macrophages from patients with COPD exhibit greater activation and elastolytic activity that those from non-COPD controls. ${ }^{28}$ Careful examination of the data revealed that A1AT deficiency favors proteolysis and tissue destruction, in general. Therefore, patients with COPD who carry inherited PiZZ A1ATD are expected to display higher levels of activated proteolytic enzymes and proteolysis-generated peptides than those with PiMM A1AT. Previous analyses of patients with COPD revealed that the protease caspase- 9 is upregulated in the lung tissue of patients with PiZZ, whereas cathepsin B is upregulated in those with PiMM. Moreover, the differences between 
PiZZ and PiMM COPD cases were found in processes related to protein biosynthesis, energy pathways and cellular defense response. ${ }^{30}$

COPD is associated with systemic abnormalities, such as renal and hormonal abnormalities, malnutrition, muscle wasting, osteoporosis and others. These systemic abnormalities have been attributed to increased systemic inflammation. Therefore, COPD is considered to have a significant systemic component. ${ }^{31}$ Urine is a rich source of biomarkers for a wide range of systemic diseases; therefore, we hypothesized that urinary peptide profiles might differ in patients with PiZZ and PiMM COPD. This is the first study to apply CS-MS for the analysis of urinary peptides in PiMM and PiZZ COPD patients.

Using CS-MS to analyze urine from patients with COPD, we detected 66 urinary peptides (corresponding to 36 unique proteins) that differed between PiMM and PiZZ cases with COPD. The peptides related to collagen breakdown products, actin organization/vesicle trafficking, lipid metabolism and the PI3K/Akt pathway are interesting candidates for distinguishing COPD with and without inherited A1ATD.

Remarkably, peptides of the MDS1 and EVI1 complex locus protein MDS1, collagen $\alpha$-2(I) chain, gelsolin and hemoglobin subunit $\alpha$, were detected in the urine of the PiZZ cases only, whereas peptides of apolipoprotein A-I, A1AT, collagen $\alpha-1$ (I) chain and hemoglobin subunit $\beta$, were detected in the urine of the PiMM cases (Table 3). Gelsolin, an actinbinding protein, is a key regulator of actin filament assembly. Caspase-3-mediated gelsolin fragmentation has been proposed to be an apoptotic effector mechanism in COPD pathogenesis and a marker of lung injury. ${ }^{32}$ Alterations in gelsolin expression have been associated with cigarette smoking and different pulmonary diseases such as fibrosis; however, little is known about the role of gelsolin in COPD. ${ }^{32-34}$ Gelsolin activates PI3K/Akt pathway, ${ }^{35}$ which regulates cell growth, proliferation, adhesion, migration and survival. Interestingly, it promotes the differentiation of alveolar epithelial type I and II cells from alveolar epithelial stem cells. ${ }^{36,37}$

COPD cases with PiZZ A1ATD presented low or undetectable levels of peptides associated with lipoproteins/ lipids and retinoic acid metabolism, such as apolipoprotein $\mathrm{A}-\mathrm{I}$ and $\mathrm{C} 4$, retinol-binding protein 4 and prostaglandin- $\mathrm{H} 2$ D-isomerase. Apolipoprotein A-I is the main component of high-density lipoproteins. Moreover, it has a protective role as an antioxidative, anti-inflammatory and antiapoptotic factor and as an inhibitor of metalloprotease activation in human lungs and cigarette smoke-exposed murine models. ${ }^{38-40}$ In addition, reduced levels of apolipoprotein A-I are observed in the lungs of patients with COPD, compared with smoker controls. ${ }^{38}$ Apolipoprotein A-I and retinol-binding protein 4 are proposed to be putative biomarkers of COPD severity. ${ }^{41,42}$

A recent review by Bidan et $\mathrm{al}^{43}$ summarized various studies on lung collagen content in COPD. Despite the fact that many studies have found increased collagen in the lungs of patients with COPD, there are contrasting data about the contribution of individual collagen subtypes. Little is known about the collagen content in urine from patients with COPD.

We identified two peptides of A1AT that were present in the urine of $43 \%$ of the PiMM cases, but in none of the PiZZ cases. A1AT and its fragments have been reported to be upregulated in chronic kidney diseases. ${ }^{44-46}$ Peptides of A1AT were detected in the urine of patients with kidney diseases and sepsis, and were suggested as putative biomarkers. ${ }^{47}$ Thus, increased urinary excretion of fragments of A1AT may reflect chronic renal damage in some COPD cases with PiMM. Further studies are required to investigate this possibility.

Due to the close relationship between the kidneys and urine, most current studies use the CE-MS method of identifying urinary biomarkers in the investigation of kidney diseases. ${ }^{48}$ However, only a limited number of urinary biomarker studies in lung diseases have been conducted, likely because the lung and the kidneys are not anatomically related, raising questions about the potential presence of lung disease biomarkers in urine. For example, enzyme-linked immunosorbent assay-based results from a recent study by Cane et al suggest that MMP levels in lung, serum and urine are independent of each other and do not reflect disease severity. ${ }^{49}$

CE-MS technology has certain limitations, as described in our previous publication. ${ }^{18}$ The small sample volume $(<200 \mu \mathrm{L})$ and inability to determine the sequence of some of the biomarker peptides are among the most relevant limitations. However, the advantages of this technology include its robustness, reproducibility and well-characterized platform, resulting in the availability of a database containing over 40,000 highly comparable individual datasets.

Use of the CS-MS method, even in a small cohort, allowed detection of distinct patterns of urinary peptides differing between the PiZZ and PiMM patients with COPD. Our novel and preliminary data suggest a clinical value for urinary peptidomics and encourage further validation of the CS-MS method in COPD patient cohorts of sufficient size for appropriate power analysis.

\section{Acknowledgment}

This study was supported by the National Science Center, Poland (grant 2015/17/B/NZ5/01370). 


\section{Disclosure}

The authors report no conflicts of interest in this work.

\section{References}

1. Halbert RJ, Natoli JL, Gano A, Badamgarav E, Buist AS, Mannino DM. Global burden of COPD: systematic review and meta-analysis. Eur Respir J. 2006;28(3):523-532.

2. Gershon AS, Warner L, Cascagnette P, Victor JC, To T. Lifetime risk of developing chronic obstructive pulmonary disease: a longitudinal population study. Lancet. 2011;378(9795):991.

3. Park TS, Lee JS, Seo JB, et al. Phenotyping of chronic obstructive pulmonary disease: heterogeneity and its clinical relevance. Curr Respir Care Rep. 2012;1(3):189-198.

4. Chorostowska-Wynimko J. Disease modification in emphysema related to alpha-1 antitrypsin deficiency. COPD. 2016;13(6):807-815.

5. Pinto-Plata V, Toso J, Lee K, et al. Use of proteomic patterns of serum biomarkers in patients with chronic obstructive pulmonary disease: correlation with clinical parameters. Proc Am Thorac Soc. 2006;3(6): 465-466.

6. Gray RD, MacGregor G, Noble D, et al. Sputum proteomics in inflammatory and suppurative respiratory diseases. Am J Respir Crit Care Med. 2008;178(5):444-452.

7. Merkel D, Rist W, Seither P, Weith A, Lenter MC. Proteomic study of human bronchoalveolar lavage fluids from smokers with chronic obstructive pulmonary disease by combining surface-enhanced laser desorption/ionization-mass spectrometry profiling with mass spectrometric protein identification. Proteomics. 2005;5(11):2972-2980.

8. Lee EJ, In KH, Kim JH, et al. Proteomic analysis in lung tissue of smokers and COPD patients. Chest. 2009;135(2):344-352.

9. Adamko DJ, Nair P, Mayers I, Tsuyuki RT, Regush S, Rowe BH. Metabolomic profiling of asthma and chronic obstructive pulmonary disease: a pilot study differentiating diseases. Allergy Clin Immunol. 2015; 136(3):571-580.

10. Magdeldin S, Hirao Y, Elguoshy A, et al. A proteomic glimpse into human ureter proteome. Proteomics. 2016;16(1):80-84.

11. Brown CE, McCarthy NS, Hughes AD, et al. Urinary proteomic biomarkers to predict cardiovascular events. Proteomics Clin Appl. 2015;9(5-6):610-617.

12. Brebner JA, Stockley RA. Recent advances in $\alpha$-1-antitrypsin deficiencyrelated lung disease. Expert Rev Respir Med. 2013;7:213-729.

13. Huang JT, Chaudhuri R, Albarbarawi O, et al. Clinical validity of plasma and urinary desmosine as biomarkers for chronic obstructive pulmonary disease. Thorax. 2012;67(6):502-508.

14. Liu X, Ma S, Liu S, Liu M, Turino G, Cantor J. The ratio of free to bound desmosine and isodesmosine may reflect emphysematous changes in COPD. Lung. 2015;193(3):329-334.

15. Mischak H, Coon JJ, Novak J, Weissinger EM, Schanstra JP, Dominiczak AF. Capillary electrophoresis-mass spectrometry as a powerful tool in biomarker discovery and clinical diagnosis: an update of recent developments. Mass Spectrom Rev. 2009;28(5):703-724.

16. Wu J, Gao Y. Physiological conditions can be reflected in human urine proteome and metabolome. Expert Rev Proteomics. 2015;12(6): 623-636.

17. Kistler AD, Serra AL, Siwy J, et al. Urinary proteomic biomarkers for diagnosis and risk stratification of autosomal dominant polycystic kidney disease: a multicentric study. PLoS One. 2013;8(1):e53016.

18. Mischak H, Kolch W, Aivaliotis M, et al. Comprehensive human urine standards for comparability and standardization in clinical proteome analysis. Proteom Clin Appl. 2010;4(4):464-478.

19. Theodorescu D, Wittke S, Ross MM, et al. Discovery and validation of new protein biomarkers for urothelial cancer: a prospective analysis. Lancet Oncol. 2006;7(3):230-240.

20. Neuhoff N, Kaiser T, Wittke S, et al. Mass spectrometry for the detection of differentially expressed proteins: a comparison of surface-enhanced laser desorption/ionization and capillary electrophoresis/mass spectrometry. Rapid Commun Mass Spectrom. 2004;18(2):149-156.
21. Dakna M, He Z, Yu WC, Mischak H, Kolch W. Technical, bioinformatical and statistical aspects of liquid chromatography-mass spectrometry (LC-MS) and capillary electrophoresis-mass spectrometry (CE-MS) based clinical proteomics: a critical assessment. J Chromatogr B Analyt Technol Biomed Life Sci. 2009;877(13):1250-1258.

22. Mischak H, Vlahou A, Ioannidis JP. Technical aspects and interlaboratory variability in native peptide profiling: the CE-MS experience. Clin Biochem. 2013;46(6):432-443.

23. Reimand J, Arak T, Adler P, et al. g:Profiler-a web server for functional interpretation of gene lists (2016 update). Nucleic Acids Res. 2016; 44(W1):W83-W89.

24. Kucich U, Christner P, Lippmann M, et al. Immunologic measurement of elastin-derived peptides in human serum. Am Rev Respir Dis. 1983; 127(2):S28-S30.

25. Wendt CH, Nelsestuen G, Harvey S, Gulcev M, Stone M, Reilly C. Peptides in bronchoalveolar lavage in chronic obstructive pulmonary disease. PLoS One. 2016;11(5):e0155724.

26. Janciauskiene $\mathrm{S}$, Welte $\mathrm{T}$. Well-Known and less well-known functions of alpha-1 antitrypsin. Its role in chronic obstructive pulmonary disease and other disease developments. Ann Am Thorac Soc. 2016;(13 Suppl 4): S280-S288.

27. Stockley RA, Burnett D. Controversy about alpha 1-antitrypsin in bronchoalveolar lavage fluids. Lancet. 1983;2(8362):1311.

28. Turino GM, Ma S, Lin YY, Cantor JO, Luisetti M. Matrix elastin: a promising biomarker for chronic obstructive pulmonary disease. $\mathrm{Am}$ J Respir Crit Care Med. 2011;184:637-641.

29. Shamamian P, Schwartz JD, Pocock BJ, et al. Activation of progelatinase A (MMP-2) by neutrophil elastase, cathepsin G, and proteinase-3: a role for inflammatory cells in tumor invasion and angiogenesis. $J$ Cell Physiol. 2001;189(2):197-206.

30. Stolk J, Seersholm N, Kalsheker N. Alpha1-antitrypsin deficiency: current perspective on research, diagnosis, and management. Int J Chron Obstruct Pulmon Dis. 2006;1(2):151-160.

31. Huertas A, Palange P. COPD: a multifactorial systemic disease. Ther Adv Respir Dis. 2011;5(3):217-224.

32. Oikonomou N, Thanasopoulou A, Tzouvelekis A, et al. Gelsolin expression is necessary for the development of modelled pulmonary inflammation and fibrosis. Thorax. 2009;64(6):467-475.

33. Bortner JD Jr, Richie JP Jr, Das A, Liao J, et al. Proteomic profiling of human plasma by iTRAQ reveals down-regulation of ITI-HC3 and VDBP by cigarette smoking. J Proteome Res. 2011;10(3):1151-1159.

34. Tang J, Ramnath N, Moysich KB, et al. Prognostic significance of MCM2, Ki-67 and gelsolin in non-small cell lung cancer. BMC cancer. 2006;6:203.

35. Huang B, Deng S, Loo SY, et al. Gelsolin-mediated activation of PI3K/ Akt pathway is crucial for hepatocyte growth factor-induced cell scattering in gastric carcinoma. Oncotarget. 2016;7(18):25391-25407.

36. Horiguchi M, Oiso Y, Sakai H, Motomura T, Yamashita C. Pulmonary administration of phosphoinositide 3-kinase inhibitor is a curative treatment for chronic obstructive pulmonary disease by alveolar regeneration. J Control Release. 2015;213:112-119.

37. Kim MN, Lee KE, Hong JY, et al. Involvement of the MAPK and PI3K pathways in chitinase 3-like 1-regulated hyperoxia-induced airway epithelial cell death. Biochem Biophys Res Commun. 2012; 421(4):790-796.

38. Kim C, Lee JM, Park SW, et al. Attenuation of cigarette smoke-induced emphysema in mice by apolipoprotein A-1 overexpression. Am J Respir Cell Mol Biol. 2016;54(1):91-102.

39. Moreno JA, Ortega-Gomez A, Rubio-Navarro A, et al. High-density lipoproteins potentiate $\alpha 1$-antitrypsin therapy in elastase-induced pulmonary emphysema. Am J Respir Cell Mol Biol. 2014;51:536-549.

40. Gordon EM, Figueroa DM, Barochia AV, Yao X, Levine SJ. Highdensity lipoproteins and apolipoprotein A-I: potential new players in the prevention and treatment of lung disease. Front Pharmacol. 2016; $7: 323$ 
41. Nicholas BL, Skipp P, Barton S, et al. Identification of lipocalin and apolipoprotein A1 as biomarkers of chronic obstructive pulmonary disease. Am J Respir Crit Care Med. 2010;181(10):1049-1060.

42. Jin Q, Chen Y, Lou Y, He X. Low Serum retinol-binding protein-4 levels in acute exacerbations of chronic obstructive pulmonary disease at intensive care unit admission is a predictor of mortality in elderly patients. J Inflamm. 2013;10(1):31.

43. Bidan CM, Veldsink AC, Meurs H, Gosens R. Airway and extracellular matrix mechanics in COPD. Front Physiol. 2015;6:346.

44. Varghese SA, Powell TB, Budisavljevic MN, et al. Urine biomarkers predict the cause of glomerular disease. J Am Soc Nephrol. 2007;18(3): 913-922.

45. Kwak NJ, Heo Y, Jin DC, et al. Proteomic analysis of alpha-1-antitrypsin in immunoglobulin A nephropathy. Proteomics Clin Appl. 2007;1(4): $420-428$.
46. Candiano G, Musante L, Bruschi M, et al. Repetitive fragmentation products of albumin and alpha1-antitrypsin in glomerular diseases associated with nephrotic syndrome. J Am Soc Nephrol. 2006;17(11): 3139-3148.

47. Blaurock N, Schmerler D, Hünniger K, et al. C-Terminal alpha-1 antitrypsin peptide: a new sepsis biomarker with immunomodulatory function. Mediators of Inflammation. 2016;2016:6129437.

48. Schanstra JP, Mischak H. Proteomic urinary biomarker approach in renal disease: from discovery to implementation. Pediatr Nephrol. 2015;30(5):713-725.

49. Cane JL, Mallia-Millanes B, Forrester DL, Knox AJ, Bolton CE, Johnson SR. Matrix metalloproteinases- 8 and -9 in the airways, blood and urine during exacerbations of COPD. COPD. 2016;13(1):26-34.

\section{Publish your work in this journal}

The International Journal of COPD is an international, peer-reviewed journal of therapeutics and pharmacology focusing on concise rapid reporting of clinical studies and reviews in COPD. Special focus is given to the pathophysiological processes underlying the disease, intervention programs, patient focused education, and self management protocols.

\section{Dovepress}

This journal is indexed on PubMed Central, MedLine and CAS. The manuscript management system is completely online and includes a very quick and fair peer-review system, which is all easy to use. Visit http://www.dovepress.com/testimonials.php to read real quotes from published authors.

Submit your manuscript here: http://www.dovepress.com/international-journal-of-chronic-obstructive-pulmonary-disease-journal 